\title{
Tolerogenic dendritic cells suppress titanium particle-induced inflammation
}

\author{
WENZHAO WANG ${ }^{1,2^{*}}$, BING HAN $^{3,4^{*}}$, JIANAN CHEN $^{3 *}$, HUICHAO TIAN $^{5}$, \\ MINGJIE SUN ${ }^{3}$, YUNPENG JIANG ${ }^{5}$ and WEI XIE ${ }^{1,2}$
}

Received June 7, 2020; Accepted March 10, 2021

DOI: $10.3892 / \mathrm{etm} .2021 .10144$

\begin{abstract}
Aseptic loosening is a major complication of prosthetic joint surgery. The leading cause of arthroplasty failure is particulate wear debris such as titanium particles. Dendritic cells (DCs) are one type of immune cells that play an important role in the initiation and progression of inflammatory processes. DCs can develop into tolerogenic DCs (tolDCs), which present an alternative therapeutic strategy for inflammatory disorders. Previously, antigen-specific tolDCs were generated, which showed a promising effect in treating inflammatory arthritis and immune thrombocytopenia. The present study reports that tolDCs effectively inhibited titanium particle-induced inflammation in an air-pouch mouse model by decreasing pro-inflammatory cytokines. In addition, a mechanistic study demonstrated that tolDCs significantly
\end{abstract}

Correspondence to: Dr Yunpeng Jiang, Department of Orthopedic, Qilu Hospital, Cheeloo College of Medicine, Shandong University, 107 West Wenhua Road, Jinan, Shandong 250012, P.R. China

E-mail: yunpeng_jiang@sina.cn

Dr Wei Xie, Department of Emergency Medicine, The Second Affiliated Hospital of Shandong First Medical University, 366 Taishan, Taian, Shandong 271000, P.R. China

E-mail:wxie@sdfmu.edu.cn

*Contributed equally

Abbreviations: TJA, total joint arthroplasty; DCs, dendritic cells; tolDCs, tolerogenic DCs; PBS, phosphate-buffered saline; ELISA, enzyme-linked immunosorbent assay; HE staining, hematoxylin and eosin staining; NOS-2, nitric oxide synthase-2; GAPDH, glyceraldehyde-3-phosphate dehydrogenase; PCR, polymerase chain reaction

Key words: tolDCs, titanium particle, inflammation, air-pouch model protected against titanium particle-induced inflammatory processes in vitro by releasing anti-inflammatory cytokines, such as interleukin-10. Collectively, these findings not only demonstrate that tolDCs play an important role in inhibiting titanium particle-induced inflammation but also provide a potential alternative for the prevention or treatment of titanium particle-induced inflammation.

\section{Introduction}

Total joint arthroplasty (TJA) is considered as one of the well-accepted surgeries to treat severe joint diseases, including rheumatoid arthritis and osteoarthritis. It effectively decreases patients' pain and improves their quality of life $(1,2)$. Approximately 1.5 million arthroplasties are performed in the world every year, and the demand for joint replacement is still increasing (3). However, revision is becoming a major concern among the complications of arthroplasty surgeries. Arthroplasty failure is mainly caused by aseptic loosening and periprosthetic osteolysis due to particulate wear debris such as titanium particles $(4,5)$. However, there is no effective clinically proven therapy or preventative drug for titanium particle-induced inflammatory osteolysis.

The types of immune cells that participate in the initiation and progression of inflammatory processes include macrophages, dendritic cells (DCs), T-helper cells and B cells $(6,7)$. DCs are antigen-presenting cells that transduce immune signals to initiate or terminate immune responses $(8,9)$. Tolerogenic dendritic cells (tolDCs) are derived from DCs in the presence of anti-inflammatory cytokines. In addition, tolDCs are thought to have the capacity to prevent or even treat some autoimmune disorders by directly interacting with antigen-specific T cells, resulting in autoimmune tolerance (10-12). For instance, tolDC has been reported to have a therapeutic effect in encephalomyelitis and pulmonary inflammation $(13,14)$. It was also found that tolDCs showed a promising effect in preventing and treating autoimmune disorders, such as inflammatory arthritis and immune thrombocytopenia (15). 
Given the importance of tolDCs in immune responses, it was hypothesized that tolDCs might represent a novel therapeutic effect for titanium-induced inflammatory osteolysis. In the present study, the role of tolDCs following the induction of titanium particle-induced inflammation was examined both in vitro and in vivo. It was found that tolDCs protected against titanium-induced inflammation by both decreasing the levels of pro-inflammatory cytokines and increasing the levels of anti-inflammatory cytokines.

\section{Materials and methods}

Media, reagents and cells. Dulbecco's Modified Eagle Medium (DMEM) and fetal bovine serum (FBS) were obtained from Gibco (Thermo Fisher Scientific, Inc.). Antibodies against nitric oxide synthase-2 (NOS-2) and glyceraldehyde-3-phosphate dehydrogenase (GAPDH) were purchased from Santa Cruz Biotechnology, Inc. Antibodies against phosphorylated (p)-P38, P38, p-P65 and P65 were purchased from Cell Signaling Technology, Inc. Enzyme-linked immunosorbent assay (ELISA) kits for tumor necrosis factor (TNF)- $\alpha$ and interleukin (IL)-1 $\beta$ were purchased from eBioscience (Thermo Fisher Scientific, Inc.). Tris, glycine, sodium dodecyl sulfate (SDS) and other reagents were obtained from Sigma-Aldrich (Merck KGaA), unless stated otherwise. RAW264.7 cells were received as a gift from Dr. Aijun Zhang's lab (Qilu Hospital, Jinan, China).

Particle preparation. Pure Ti particles were obtained from Johnson Matthey Chemicals. Ti particles were prepared by autoclaving, followed by washing in $100 \%$ alcohol 3 times. The endotoxin was then detected and removed using the Limulus Amebocyte Lysate kit as described previously $(16,17)$.

Generation, cultivation and identification of tolDCs. Bone marrow-derived dendritic cells (BmDCs) were isolated and cultured in the presence of $10 \mathrm{ng} / \mathrm{ml}$ granulocyte-macrophage colony-stimulating factor (GM-CSF) and $1 \mathrm{ng} / \mathrm{ml} \mathrm{IL-4,} \mathrm{as}$ previously described (18). TolDCs were generated by culturing BmDCs in the presence of $20 \mathrm{ng} / \mathrm{ml} \mathrm{IL-10} \mathrm{and} 20 \mathrm{ng} / \mathrm{ml}$ transforming growth factor- $\beta$ for 6 days. TolDCs were purified using anti-CD11c microbeads. To determine the phenotype of generated tolDCs, flow cytometry was performed by staining cell surface markers, including FITC-labeled CD11c and major histocompatibility complex (MHC)-II and phycoerythrin-conjugated CD80, as well as CD86 (Biolegend, Inc.). DCs showed CD11 positivity.

Animals and titanium particles-stimulated mouse air-pouch model. The Institutional Animal Care and Use Committee of Jinan Central Hospital and Qilu Hospital approved all animal studies. All procedures were performed in accordance with institutional guidelines. BALB/c mice (8-10 weeks old) were obtained from Shandong University. The animals were housed in a clean facility under the controlled conditions of $22-24^{\circ} \mathrm{C}$, relative humidity of $30-50 \%$, and a 12 -h light-dark cycle. A standard mouse diet and filtered water were available ad libitum.

Titanium particle-induced (Ti-induced) air pouches were generated according to a previously described method $(19,20)$. Mice were anesthetized with $3 \%$ pentobarbital $(30 \mathrm{mg} / \mathrm{kg}$, ip).
The dorsal skins of the mice were cleaned and shaved before surgery. A sterilized air-pouch was prepared by subcutaneous injection of $3 \mathrm{ml}$ sterilized syringe. On the second day, pouches were initiated to provoke inflammation by injecting $0.5 \mathrm{ml}$ PBS diluted with $5 \mathrm{mg}$ Ti particles. In addition, the air pouches were maintained by injecting $1 \mathrm{ml}$ air every other day until day 5 . The mice were randomly divided into two groups, each group comprised 10 mice. For the control group, $0.5 \mathrm{ml}$ PBS was injected daily into pouches. For the mice with treatment, tolDCs $\left(1 \times 10^{6}\right)$ were injected into pouches daily. Mice were humanely sacrificed by cervical dislocation on day 12 . Cervical dislocation euthanasia was preformed after the mouse was anesthetized with $3 \%$ pentobarbital (30 mg/kg, ip). The thumb and index finger of one hand pressed the head of the mouse, and the other hand grasped the tail, quickly and forcefully pulled backward and upward to dislocate the cervical vertebra. A slight sense of absence could be felt when the cervical vertebra was dislocated. The mouse lost its vital signs in an instant. No experimental animal died during the experiment. The pouch membranes were harvested for further analysis.

RAW 264.7 cell culture and stimulation. RAW 264.7 cells were cultured in DMEM containing $10 \% \mathrm{FBS}$ at $37^{\circ} \mathrm{C}$ in a humidified incubator with $5 \% \mathrm{CO}_{2}$. To investigate the effects of particle stimulation on an array of mRNA gene transcripts, RAW 264.7 cells were cultured in the absence or presence of tolDC cultured medium with $1 \%$ Ti particles dissolved in the same medium for $6 \mathrm{~h}$ before RNA extraction. Protein was extracted after 24 and 48 h of Ti particle stimulation.

Histology. After harvesting the skin membrane, it was fixed in $4 \%$ paraformaldehyde for $24 \mathrm{~h}$ at room temperature and then embedded it in olefin. At least 5 consecutive $6-\mu \mathrm{m}$ sagittal sections were collected for staining. Sections were stained using hematoxylin and eosin (HE; cat. no. C0105S Beyotime Institute of Biotechnology) and Masson's Trichrome (cat. no. G1340; Beijing Solarbio Science \& Technology Co., Ltd.). Images were acquired with an inverted optical microscope (magnification, x10; Olympus, Corporation).

Reverse transcription-quantitative $(R T-q) P C R$. Total RNA was extracted from RAW 264.7 cells or skin using an RNeasy kit (Qiagen, Inc.). Reverse transcription was performed using an RT-for-PCR kit (Qiagen, Inc.). The expression levels of mRNAs were measured by qPCR with QuantiTect SYBR Green RT-PCR Kit (Qiagen, Inc.) using a 7500 RT-PCR system (Applied Biosystems; Thermo Fisher Scientific, Inc.). The thermocycling conditions were as follows: Initial denaturation at $95^{\circ} \mathrm{C}$ for $10 \mathrm{~min}$, followed by 40 cycles of denaturation at $95^{\circ} \mathrm{C}$ for $15 \mathrm{sec}$, annealing at $60^{\circ} \mathrm{C}$ for $30 \mathrm{sec}$ and elongation at $72^{\circ} \mathrm{C}$ for $1 \mathrm{~min}$, followed by final elongation at $72^{\circ} \mathrm{C}$ for $2 \mathrm{~min}$. All these procedures were performed according to the manufacturer's protocol. The primers for qPCR used in the present study are listed as follows: IL- $1 \beta$ forward, 5'-AATCTC ACAGCAGCACATCA-3'; IL-1 $\beta$ reverse, 5'-AAGGTGCTC ATGTCCTCATC-3'; IL-6 forward, 5'-ATGAAGTTCCTC TCTGCAAGAGACT-3'; IL-6 reverse, 5'-CACTAGGTTTGC CGAGTAGATCTC-3'; cyclooxygenase (COX)-2 forward, 5'-AATGCTGACTATGGCTACAAAA-3'; COX-2 reverse, 
5'-AAAACTGATGCGTGAAGTGCTG-3'; NOS-2 forward, 5'-CAGCCTCTGTCTCTCAGGCTCTT-3'; NOS-2 reverse, 5'-CTCTCTAAGTGA ACAACTGGCCTGTGA-3'; IL-10 forward, 5'-ACCTGGTAGAAGTGATGCC-3'; IL-10 reverse, 5'-CAAGGAGTTGTTTCCGTTA-3'; GAPDH forward, 5'-ACCCAGAAGACTGTGGATGG-3'; GAPDH reverse, 5'-CACATTGGGGGTAGGAACAC-3'. The mRNA expression was normalized to that of GAPDH. The value $2^{-\Delta \Delta C q}$ was used for comparative quantitation (15). Each experiment was repeated at least three times.

Western blotting. Total air-pouch membranes and RAW 264.7 cell extracts were homogenized and proteins were collected using protein extraction buffer (cat. no. P0013; Beyotime Institute of Biotechnology). Protein concentrations in the supernatant were detected using the enhanced BCA Protein Assay kit (Beyotime Institute of Biotechnology). The supernatant samples containing $1 \mathrm{mg}$ protein $/ 500 \mu \mathrm{l}$ were resolved on 8\% SDS-polyacrylamide gel and electroblotted onto a nitrocellulose membrane. BSA (3\%) was diluted in Tris-buffered saline-Tween 20 (TBST; $10 \mathrm{mM}$ Tris- $\mathrm{HCl}, \mathrm{pH} 8.0 ; 150 \mathrm{mM}$ $\mathrm{NaCl}$; and $0.5 \%$ Tween 20 ) and incubated with the membrane for $1 \mathrm{~h}$ at room temperature. The membrane was then incubated at $4^{\circ} \mathrm{C}$ with anti-NOS-2 (1:1,000; cat. no. sc-649, $120 \mathrm{kDa}$; Santa Cruz Biotechnology, Inc.), anti-p-P38 (1:1,000, cat. no. 4511, $43 \mathrm{kDa}$; Cell Signaling Technology, Inc.), anti-P38 (1:1,000; cat. no. 8690; 40 kDa, Cell Signaling Technology, Inc.), anti-p-P65 (1:1,000, cat. no. 3033, 65 kDa; Cell Signaling Technology, Inc.), anti-P65(1:1,000, cat. no. 8242; 65 kDa; Cell Signaling Technology, Inc.), or anti-GAPDH (1:1,000, cat. no. 25778; $36 \mathrm{kDa}$; Santa Cruz Biotechnology, Inc.) primary antibodies overnight. After washing 3 times with TBST (each $10 \mathrm{~min}$ ), the secondary antibody (HRP-conjugated anti-rabbit immunoglobulin; GB23303, 1:5,000, Wuhan Servicebio Technology Co., Ltd.) was added and incubated at room temperature for $1 \mathrm{~h}$, and the bound antibody was visualized using West Pico typically enhanced chemiluminescent Substrate (Beijing Solarbio Science \& Technology Co., Ltd.).

ELISA. The levels of pro-inflammatory cytokines in the sera or cultured medium were assessed using ELISA kits (IL-6, cat. no. 88-7064; IL-10, cat. no. 88-7105; TNF- $\alpha$, cat. no. 88-7324; IL-1 $\beta$, cat. no. 88-7013; eBioscience; Thermo Fisher Scientific, Inc.), as previously described (21). An ELISA reader (Molecular Devices, LLC) at $450 \mathrm{~nm}$ was used to determine the optical intensity. In addition, the concentration of the examined cytokines were analyzed by regression against a generated standard curve.

Statistical analysis. Statistical analysis was performed using GraphPad Prism software 8.0.2 (GraphPad Software, Inc.). Data are expressed as means \pm deviation (SD). Unpaired-samples t-test was used to assess statistical significance. $\mathrm{P}<0.05$ was considered to indicate a statistically significant difference.

\section{Results}

TolDCs sustain immune tolerogenic phenotype. To confirm the phenotype and function of the generated tolDCs, cell surface markers were identified by cytometry assay, including CD11c,
CD80, MHC-II and CD86. As demonstrated in Fig. 1A-D, compared with naïve DCs, the expression of these markers in tolDCs was dramatically decreased. To further confirm this finding, mRNA was extracted and RT-qPCR analysis was performed. As shown in Fig. 1E and F, the transcriptional levels of IL-12 and C-C chemokine receptor type 7 (CCR7) were significantly decreased in tolDCs. In addition, as shown in Fig. 1G, the expression of the anti-inflammatory cytokine IL-10 was significantly increased in tolDCs. Collectively, tolDCs presented not only a tolerogenic phenotype but also an anti-inflammatory effect.

TolDCs suppress the Ti-induced inflammatory phenotype in the mouse air-pouch model. To determine whether tolDCs could prevent Ti-induced inflammation in vivo an air-pouch model was established in BALB/c mice and PBS or tolDCs were injected daily until the mice were sacrificed. As shown in Fig. 2A-C, HE staining showed that the thickness of the membrane in the tolDCs-treated group was significantly decreased compared with that of the PBS control group. In addition, Fig. 2B revealed that Masson's trichrome staining also validated this finding, showing that tolDCs prevented inflammatory swelling; which was further confirmed by statistical analysis of the membrane thickness, as illustrated in Fig. 2C. These in vivo data indicated that tolDCs effectively inhibited the Ti-induced inflammatory phenotype.

TolDCs decreases inflammatory and enhances anti-inflammatory gene expression in the air-pouch membrane. To further determine whether gene expression changes in the Ti-induced skin membrane, mice were sacrificed on day 6 and collected the skin membrane, followed by RNA extraction. TNF- $\alpha$ is known to play an important role in the Ti-induced inflammatory process. To determine the anti-inflammatory effect of tolDCs, the levels of pro-inflammatory markers such as TNF- $\alpha$, IL-1 $\beta$, IL-6, NOS-2 and COX-2 were measured. As shown in Fig. 3A-E, all these pro-inflammatory markers were dramatically decreased by the additional use of tolDCs compared with those in the PBS-treated group. In addition, it is well accepted that tolDCs secrete IL-10, which plays a critical anti-inflammatory role. As illustrated in Fig. 3F, IL-10 gene expression was significantly increased. Collectively, tolDCs effectively lowered pro-inflammatory gene expression and enhanced anti-inflammatory gene expression in the Ti particle-induced skin membrane.

TolDCs decreased Ti-induced inflammation. To further confirm the present findings, the skin membranes were collected and western blotting was performed for the expression of NOS-2, which is regarded as an indicator of inflammatory severity. As shown in Fig. 4A, tolDCs dramatically decreased NOS-2 protein expression, indicating that tolDCs decreased inflammatory severity. In addition, sera was collected after sacrificing the mice and ELISA was performed for TNF- $\alpha$, IL-1 $\beta$, and IL-6 expression levels. As shown in Fig. 4B-D, all these pro-inflammatory cytokines were significantly decreased in the tolDCs-treated group. In contrast, the expression of the anti-inflammatory cytokine IL-10 was significantly increased after the additional use of tolDCs (Fig. 4E). Overall, tolDCs effectively treated Ti particle-induced inflammation both locally and systematically. 
A
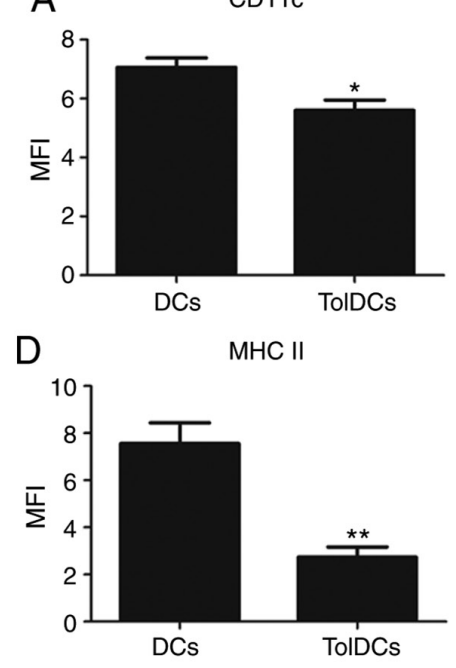

B

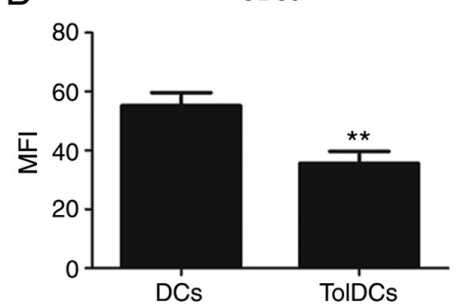

E

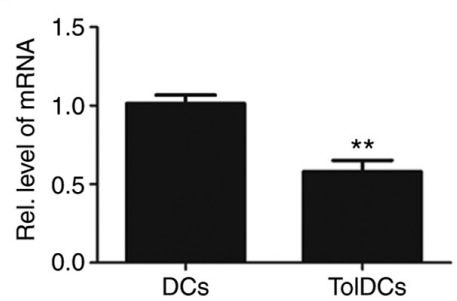

C

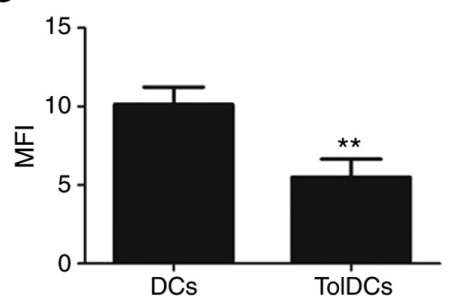

$\mathrm{F}$

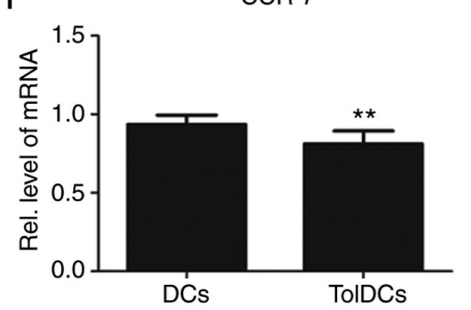

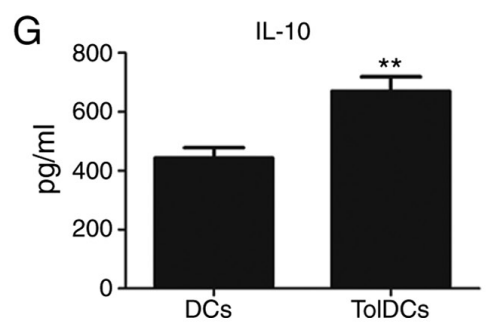

Figure 1. TolDCs sustain immune tolerogenic phenotype. (A-D) Comparison of cell surface markers such as CD11c, MHC-II, CD80 and CD86 in DCs and tolDCs, analyzed by flow cytometry assay. (E and F) Transcriptional level of IL-12 and CCR7 in DCs and tolDCs, analyzed by reverse transcription-quantitative PCR assay. (G) Enzyme linked immunosorbent assay for determining IL-10 protein levels in DC culture media. " $\mathrm{P}<0.05$; ${ }^{* *} \mathrm{P}<0.01$. MFI, median fluorescence intensity; DCs, dendritic cells; TolDCs, tolerogenic DCs; MHC, major histocompatibility complex; IL, interleukin; CCR7, C-C chemokine receptor type 7.

A

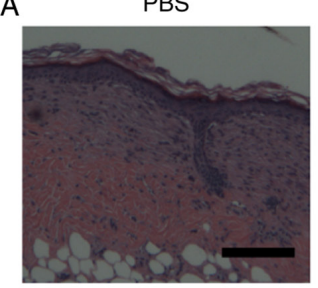

B
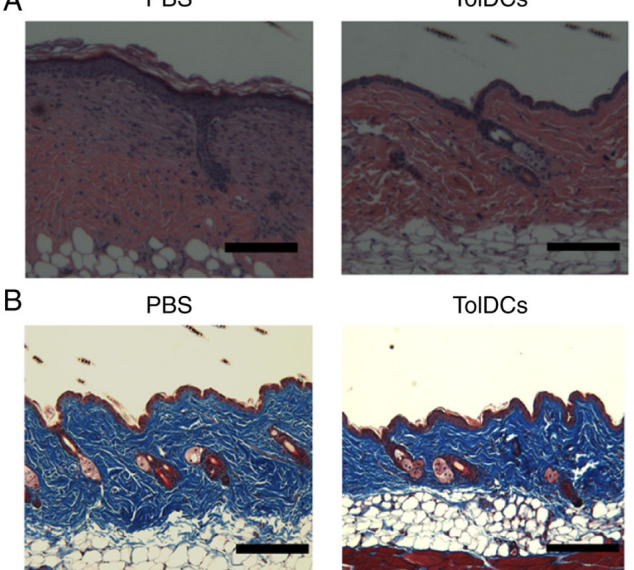

TolDCs

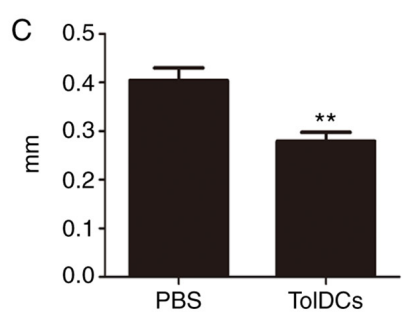

Figure 2. TolDCs suppress Ti-induced inflammatory phenotype in mouse air-pouch model. (A) HE staining of air-pouch membrane. Scale bar, $100 \mu \mathrm{m}$. (B) Masson Trichrome staining of air-pouch membrane. Scale bar, $100 \mu \mathrm{m}$. (C) Statistic analysis of skin thickness in both PBS-treated and tolDCs-treated mice according to HE staining. $\left(\mathrm{n}=6 ;{ }^{* * *} \mathrm{P}<0.01\right)$. TolDCs, tolerogenic dendritic cells; HE, hematoxylin and eosin.
TolDCs suppress Ti-induced inflammatory signaling in vitro. Given the importance of tolDCs-mediated anti-titanium-induced inflammation in vivo, primary bone marrow cells were cultured to determine the molecular mechanisms involved. Briefly, tolDCs were cultured in regular medium for 3 days and then collected the supernatant for further study. The collected medium was used to treat primary bone marrow cells in the presence of $1 \%$ titanium particles for different time points. As illustrated in Fig. 5, titanium promotes the phosphorylation of $\mathrm{P} 35$ and $\mathrm{P} 65$, but tolDCs inhibit this process.

TolDCs suppress Ti-induced inflammation by decreasing the expression of pro-inflammatory cytokines and enhancing the expression of anti-inflammatory cytokines in vitro. To further determine the role of tolDCs in Ti-induced inflammation, tolDCs were cultured in regular medium for 3 days and the supernatant was then collected for further study. The tolDC medium was diluted with regular medium (RM) at a ratio of 1:3 to make the conditional medium $(\mathrm{CM})$. The $\mathrm{CM}$ or RM was used to culture RAW264.7 cells in the presence of $1 \%$ titanium particles for $6 \mathrm{~h}$. Next, mRNA was extracted and a RT-qPCR assay was performed. As indicated in Fig. 6A-D and F, the gene expression of pro-inflammatory cytokines such as TNF- $\alpha$, IL- $1 \beta$, IL- 6 , NOS- 2 and COX- 2 was notably decreased in the $\mathrm{CM}$ treated group, indicating that tolDCs strongly inhibit Ti-induced inflammation. To further extend this finding, RAW 264.7 cells were cultured with CM or RM in the presence of $1 \%$ titanium particles for 3 days. 

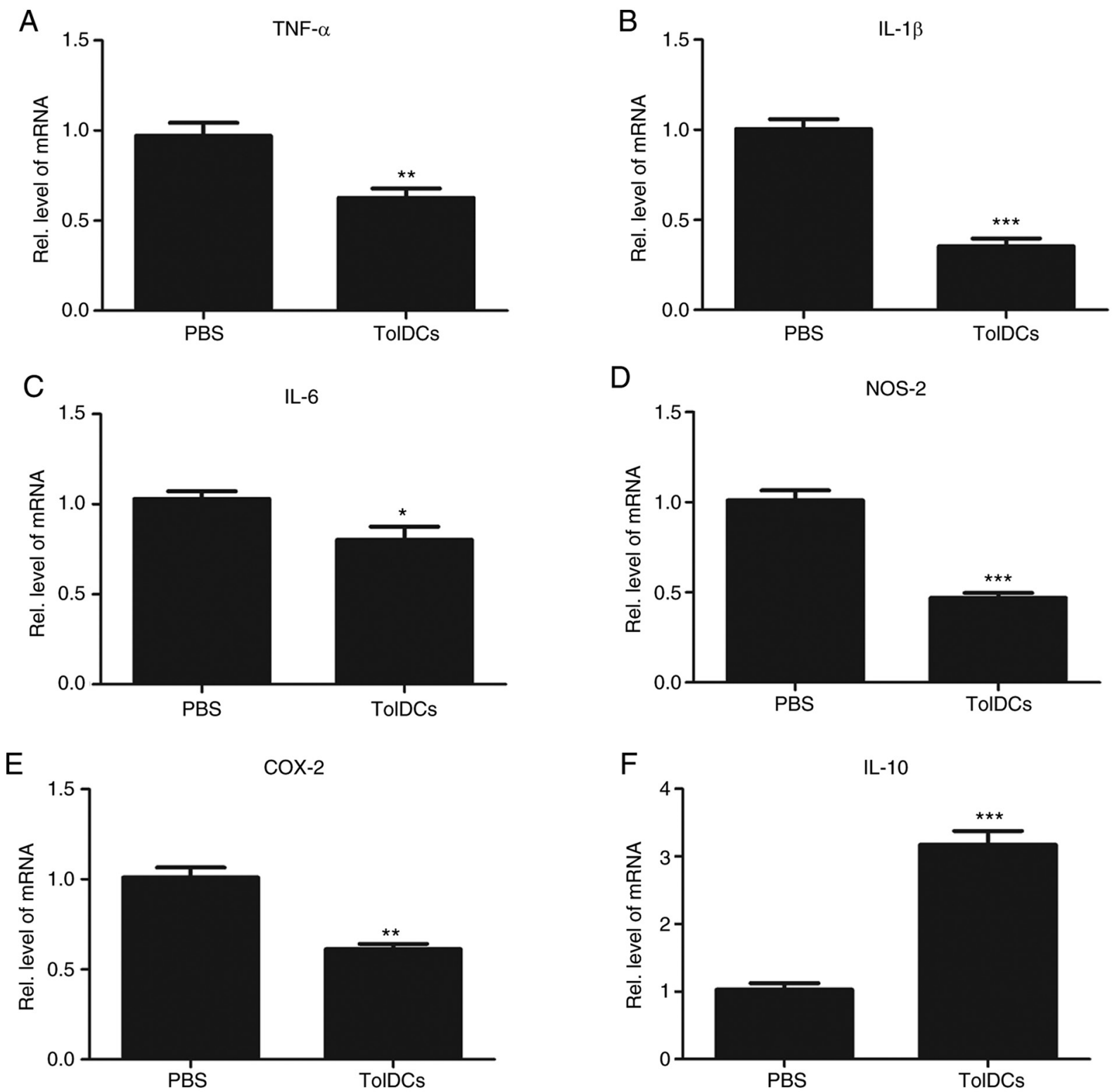

Figure 3. TolDCs decreases inflammatory gene expression and enhances anti-inflammatory gene expression in air-pouch membrane. Transcriptional levels of pro-inflammatory mediators including (A) TNFa, (B) IL-1 $\beta$, (C) IL-6, (D) NOS-2 and (E) COX-2, and anti-inflammatory mediators (F) IL-10 in PBS- and tolDCs-treated mice skin measured by RT-qPCR. The units were arbitrary, and the normalized values were calibrated against the PBS control, and each RT-qPCR was performed at least in triplicate. Values are the normalized mean $\pm \mathrm{SEM} .{ }^{*} \mathrm{P}<0.05 ;{ }^{* * *} \mathrm{P}<0.01 ;$ and ${ }^{* * * *} \mathrm{P}<0.001$. Ten mice were used in each group. TolDCs, tolerogenic dendritic cells; TNF $\alpha$, tumor necrosis factor- $\alpha$; IL, interleukin; COX, cyclooxygenase; NOS-2, nitric oxide synthase-2; RT-qPCR, reverse transcription-quantitative PCR; Rel., relative.

Then, supernatant was collected for ELISA analysis. As shown in Fig. 6G-I, the release of pro-inflammatory cytokines, including TNF- $\alpha$, IL- $1 \beta$ and IL- 6 , was dramatically decreased in the CM. In contrast, as shown in Fig. $6 \mathrm{E}$ and J, the expression of the anti-inflammatory cytokine IL-10 was significantly increased in the CM-treated group. Collectively, tolDCs could suppress Ti-induced inflammation by decreasing the levels of pro-inflammatory cytokines and increasing the levels of anti-inflammatory cytokines.

\section{Discussion}

Aseptic loosening may cause periprosthetic osteolysis, resulting in TJA failure and revision surgery. It is known that prosthetic loosening is primarily driven by wear debris-mediated inflammatory osteolysis and has been shown to involve titanium particles (22-24). The air-pouch model exhibits cellular infiltration and mediators of inflammation that appear to closely resemble the pseudo synovium associated with aseptic loosening (19,20). Marked responses to titanium particles were observed, the particles deeply embedded within the tissue and surrounded by macrophages. This model accurately simulates the physiological state of peri-prosthetic tissue.

Dendritic cells (DCs) are a type of well-known immune cells that present antigens for the induction of immune response. Based on the properties of DCs, many worldwide labs generated tolDCs independently and they confirmed the autoimmune tolerance effect (6). Given the importance of tolDCs in the immune system, more and more findings indicated that tolDCs would be a promising cellular immunotherapeutic agent for 

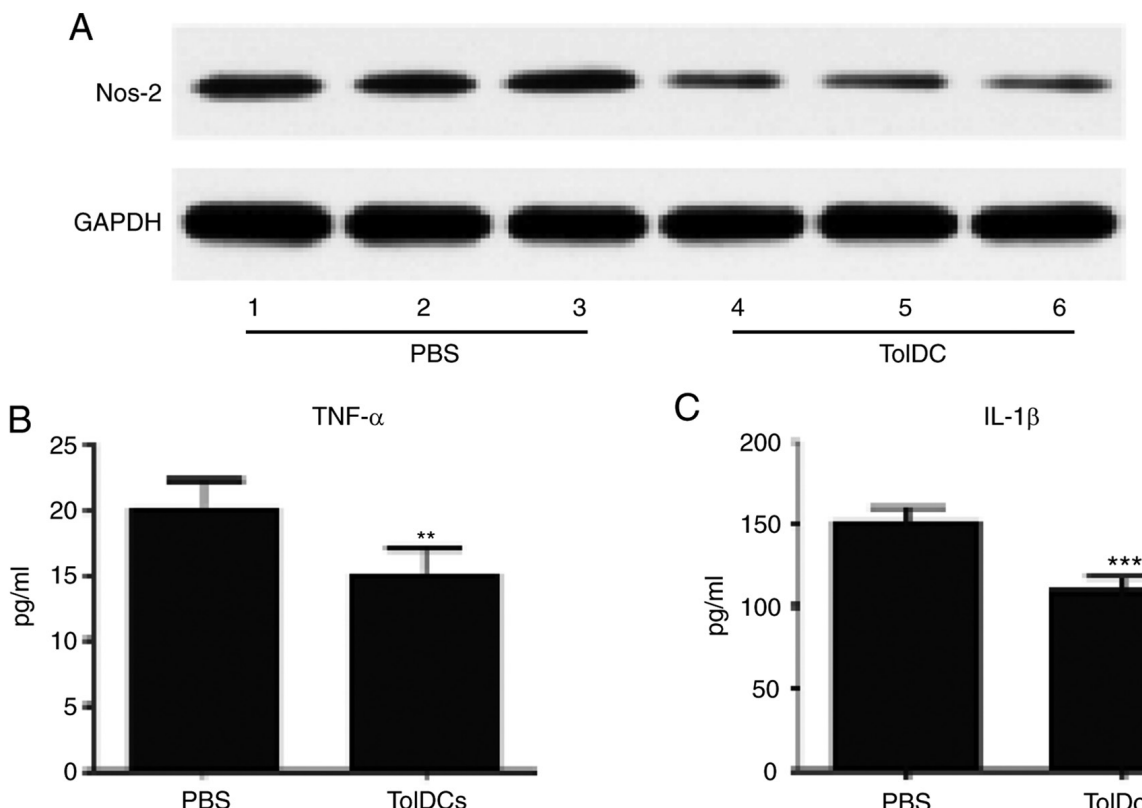

C
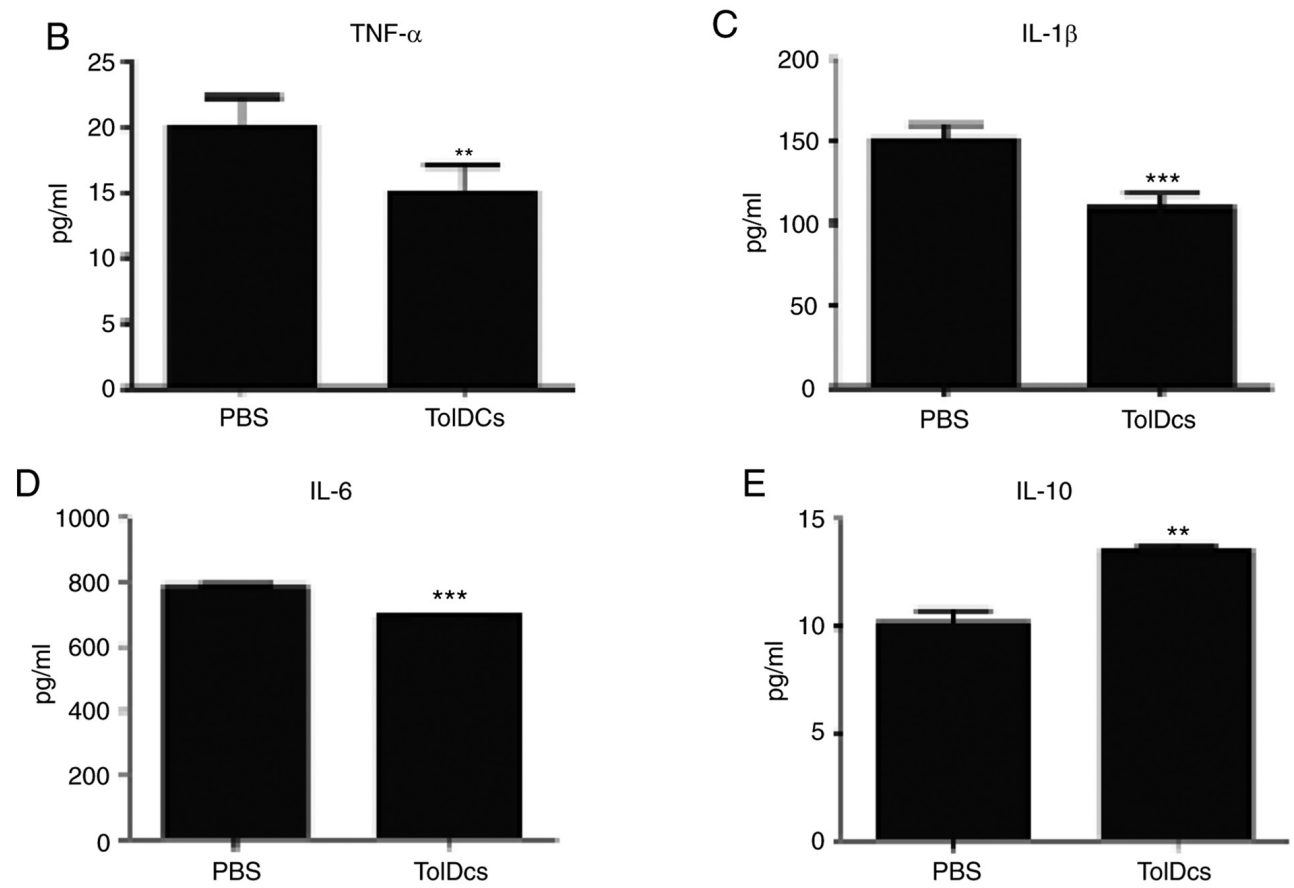

Figure 4. TolDCs locally and systematically decreased titanium-particle-induced inflammation. (A) Expression of NOS-2 in control PBS- and tolDCs-treated titanium particle-induced mice air-pouch membrane detected by western blotting. (B-D) Serum levels of the pro-inflammatory cytokines TNF $\alpha$, (B) IL-1 $\beta$ (C) and IL-6 (D) in PBS- and tolDCs-treated mice in titanium particle-stimulated air-pouch model, assayed by ELISA. (E) Serum levels of the anti-inflammatory cytokine IL-10 in PBS- and tolDC-treated mice in titanium particle-stimulated air-pouch model assayed by ELISA. Values are the normalized mean \pm SEM ${ }^{* *} \mathrm{P}<0.01$; and ${ }^{* * *} \mathrm{P}<0.001$. Six mice were used in each group. TolDCs, tolerogenic dendritic cells; TNF $\alpha$, tumor necrosis factor- $\alpha$; IL, interleukin; NOS-2, nitric oxide synthase-2; ELISA, enzyme-linked immunosorbent assay.

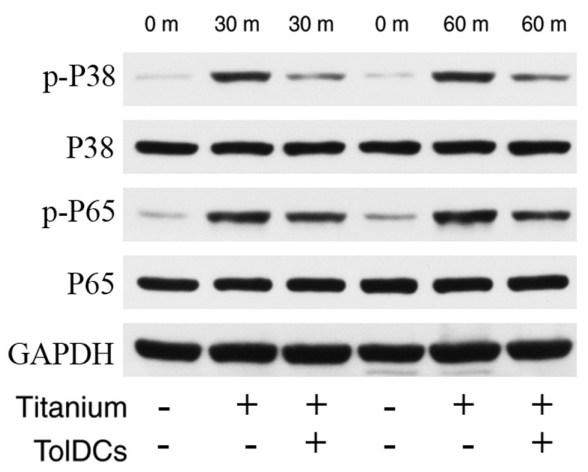

Figure 5. TolDCs suppress titanium particle-induced inflammatory signaling. Primary bone marrow cells were cultured with or without titanium particles in the absence or presence of the TolDCs-cultured medium. Protein was extracted at different time points, followed by western blot assay. TolDCs, tolerogenic dendritic cells; p-, phosphorylated.

some inflammatory diseases. Furthermore, it was shown that tolDCs could induce platelet-specific immune tolerance (25). In addition, antigen-specific tolDCs that were generated showed an effective therapeutic effect in inflammatory arthritis. The present study extended the previous findings that tolDCs were therapeutic in Ti-induced inflammation.

TolDCs are known to have anti-inflammatory activity under various conditions. In the present study, it was found that tolDCs could significantly suppress Ti-induced inflammation in a mouse air-pouch model, which implied that tolDCs might also play an anti-inflammatory role in wear debris-mediated pathological processes. Indeed, tolDCs decreased the Ti-induced inflammatory response, as indicated by the phenotype and decreased levels of pro-inflammatory cytokines such as TNF $\alpha$, IL-1 $\beta$ and IL- 6 , both at the mRNA expression and protein synthesis levels. In addition, tolDCs effectively prevent Ti-induced inflammation. COX-2 has been recognized as an inducible or pathological enzyme and participates in the inflammatory response by facilitating prostaglandin synthesis (26). The NOS-2 level was elevated in the joint interface membrane tissues of arthritis, and suppression of NOS-2 indicated alleviation of inflammation (27). In the present study, it was found that COX-2 and NOS-2 levels were enhanced by titanium particles, a finding that is consistent with a previous publication (28). This Ti-induced COX-2 and NOS-2 expression was markedly inhibited by tolDCs. From the 
A

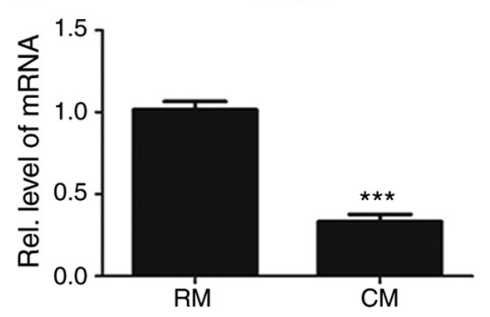

D

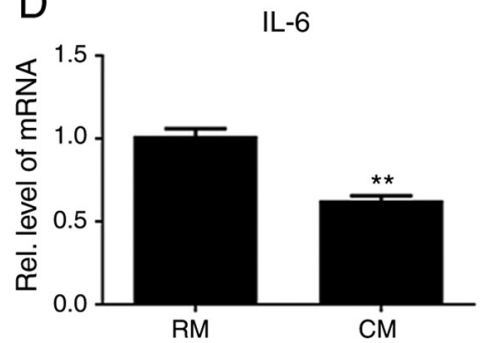

$\mathrm{G}$

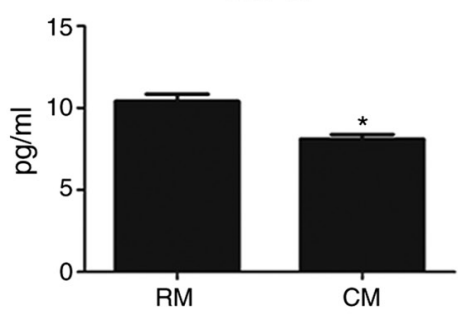

B

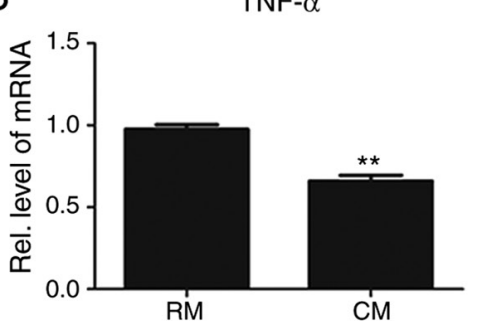

$\mathrm{E}$

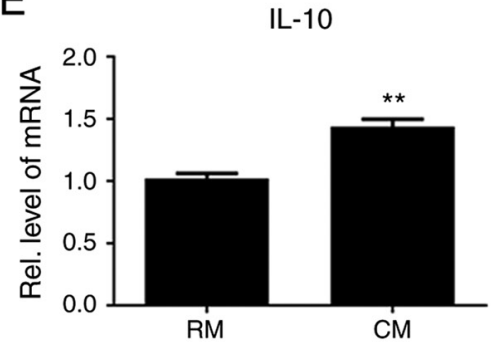

$\mathrm{H}$

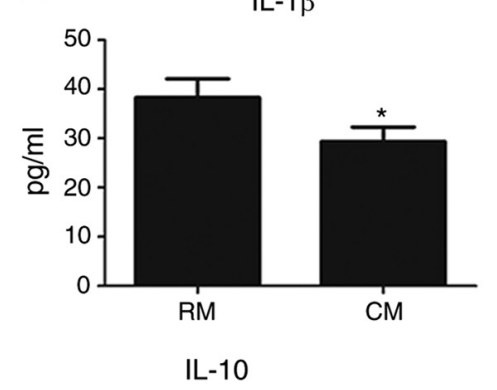

C

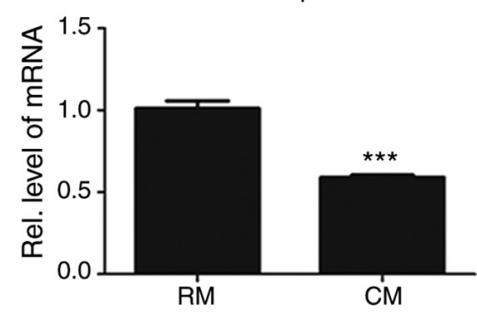

$\mathrm{F}$

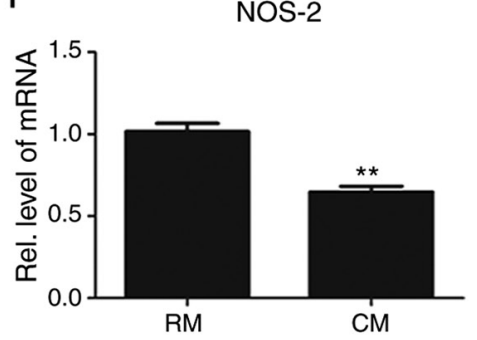

I

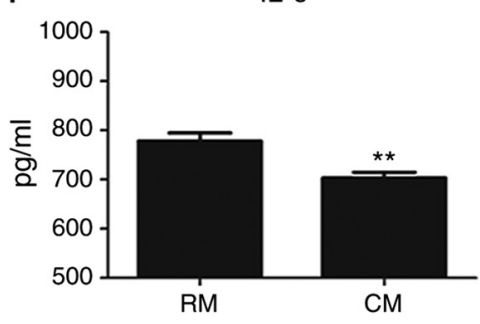

$J$

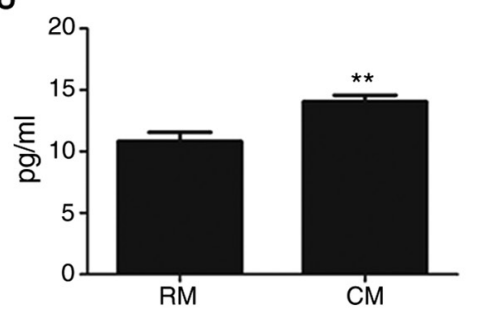

Figure 6. TolDCs suppress titanium particle-induced inflammation in vitro. (A) COX-2, (B) TNF- $\alpha$, (C) IL-1 $\beta$, (D) IL-6 and (F) NOS-2 from RAW264.7 cells with RM or CM in the presence of titanium, measured by RT-qPCR assay. The units were arbitrary, and the normalized values were calibrated against the PBS control group, and each RT-qPCR was performed at least in triplicate. (E) Levels of anti-inflammatory cytokine IL-10 in RM or CM in titanium particle-stimulated RAW 264.7 cells assayed by RT-qPCR. (G-I) Levels of the pro-inflammatory cytokines (G) TNF- $\alpha$, (H) IL-1 $\beta$, and (I) IL-6 in RM or CM in titanium particle-stimulated RAW 264.7 cells assayed by ELISA. (J) Levels of the anti-inflammatory cytokine IL-10 in RM or CM in titanium particle-stimulated RAW 264.7 cells assayed by ELISA. Values are the normalized mean \pm SEM. ${ }^{*} \mathrm{P}<0.05 ;{ }^{* * *} \mathrm{P}<0.01$; and ${ }^{* * * *} \mathrm{P}<0.001$. TolDCs, tolerogenic dendritic cells; RM, regular medium; CM, conditional medium; RT-qPCR, reverse transcription-quantitative PCR; ELISA, enzyme-linked immunosorbent assay; TNFa, tumor necrosis factor- $\alpha$; IL, interleukin; COX, cyclooxygenase; NOS-2, nitric oxide synthase-2.

aforementioned, various inflammatory factors are regulated by TolDCs, and the present study preliminarily detected and verified the associated signaling pathways. The nuclear factor (NF)- $\mathrm{kB}$ and MAPK signaling pathways regulate numerous cellular activities, particularly in arthritis-associated inflammation $(24,27)$. The results showed that titanium dramatically activated P38 MAPK and NF-кB P65 signaling. In contrast, the $\mathrm{CM}$ from tolDCs effectively prevented the activation of these pro-inflammatory pathways. Thus, tolDCs could inhibit Ti-induced pro-inflammatory pathways.

To better understand the role of tolDCs in Ti-induced inflammation, tolDCs were cultured in RM for 3 days. Subsequently, the medium was collected and mixed with the $\mathrm{RM}$ at a ratio of 1:3 to make the $\mathrm{CM}$. It was found that the $\mathrm{CM}$ strongly suppressed Ti-induced pro-inflammatory cytokine expression at both the gene and protein levels. This finding suggests that tolDCs might secrete anti-inflammatory cytokines, such as IL-10, to protect against Ti-induced inflammation. To identify these possible cytokines, further studies need to be performed. The present study also has the following limitations: Histological staining for animal modeling was lacking in this study; this study has preliminarily verified that tolDCs regulated the expression of COX-2, NOS-2, TNF $\alpha$, IL-1 $\beta$, IL- 6 and IL-10 through the $\mathrm{NF}-\mathrm{KB}$ and MAPK signaling pathways. However, the specific target is still unknown. In future studies, the specific target and mechanism of Ti-induced inflammation should be explored.

Based on the findings in the present study, as well as previous reports, it was found that titanium particles lead to the release of pro-inflammatory cytokines such as TNF- $\alpha$, IL-1 $\beta$ and IL-6. However, local injection of tolDCs prevents 
Ti-induced inflammatory pathogenesis by decreasing pro-inflammatory cytokines and increasing anti-inflammatory cytokines. Collectively, the findings reported in the present study not only provide new insights into the molecular mechanisms underlying Ti-induced inflammation but may also present tolDCs as a new therapeutic strategy for the prevention of aseptic loosening.

\section{Acknowledgements}

The authors wish to acknowledge Ms Yutian Huang (Department of Education, University of Sheffield) for her guidance on language.

\section{Funding}

This study was funded by Shandong Medical and Health Science and Technology Development Programs (grant no. 2016WS0618); and The Innovation and Entrepreneurship Project of Sichuan Technology Gallery, Sichuan Science and Technology Program, China (grant no. 2020JDRC0054).

\section{Availability of data and materials}

The datasets used and/or analyzed during the current study are available from the corresponding author on reasonable request.

\section{Authors' contributions}

WW, BH and JC carried out the animal model design and the molecular study. HT and MS participated in the data analysis. YJ and WX conceived the study, participated in its design and coordination, and helped to draft the manuscript. YJ and WX confirm the authenticity of all the raw data. All authors read and approved the final manuscript.

\section{Ethics approval and consent to participate}

The Institutional Animal Care and Use Committee of Qilu Hospital approved all animal studies (approval no. KYLL-2019(KS)-028). All procedures were performed in accordance with institutional guidelines.

\section{Patient consent for publication}

Not applicable.

\section{Competing interests}

The authors declare that they have no competing interests.

\section{References}

1. Tian B, Jiang T, Shao Z, Zhai Z, Li H, Fan Q, Liu X, Ouyang Z, Tang T, Jiang Q, et al: The prevention of titanium-particle-induced osteolysis by OA-14 through the suppression of the p38 signaling pathway and inhibition of osteoclastogenesis. Biomaterials 35: 8937-8950, 2014.

2. Wei J, Liu CJ and Li Z: ADAMTS-18: A metalloproteinase with multiple functions. Front Biosci (Landmark Ed) 19: 1456-1467, 2014.
3. Teeny SM, York SC, Mesko JW and Rea RE: Long-term follow-up care recommendations after total hip and knee arthroplasty: Results of the American Association of Hip and Knee Surgeons' member survey. J Arthroplasty 18: 954-962, 2003.

4. Harris WH: Wear and periprosthetic osteolysis: The problem. Clin Orthop Relat Res 393: 66-70, 2001.

5. Dumbleton JH, Manley MT and Edidin AA: A literature review of the association between wear rate and osteolysis in total hip arthroplasty. J Arthroplasty 17: 649-661, 2002.

6. Zhao Y, Zhang A, Du H, Guo S, Ning B and Yang S: Tolerogenic dendritic cells and rheumatoid arthritis: Current status and perspectives. Rheumatol Int 32: 837-844, 2012.

7. Landgraeber S, Jäger M, Jacobs JJ and Hallab NJ: The Pathology of orthopedic implant failure is mediated by innate immune system cytokines. Mediators Inflamm 2014: 185150, 2014.

8. Piccioli D, Tavarini S, Borgogni E, Steri V, Nuti S, Sammicheli C, Bardelli M, Montagna D, Locatelli F and Wack A: Functional specialization of human circulating CD16 and CD1c myeloid dendritic-cell subsets. Blood 109: 5371-5379, 2007.

9. Xie ZX, Zhang HL, Wu XJ, Zhu J, Ma DH and Jin T: Role of the immunogenic and tolerogenic subsets of dendritic cells in multiple sclerosis. Mediators Inflamm 2015: 513295, 2015.

10. Oriss TB, Ostroukhova M, Seguin-Devaux C, Dixon-McCarthy B, Stolz DB, Watkins SC, Pillemer B, Ray P and Ray A: Dynamics of dendritic cell phenotype and interactions with CD4+ T cells in airway inflammation and tolerance. J Immunol 174: 854-863, 2005.

11. Wang L, Pino-Lagos K, de Vries VC, Guleria I, Sayegh MH and Noelle RJ: Programmed death 1 ligand signaling regulates the generation of adaptive Foxp3+CD4+ regulatory T cells. Proc Natl Acad Sci USA 105: 9331-936, 2008.

12. Morelli AE and Thomson AW: Tolerogenic dendritic cells and the quest for transplant tolerance. Nat Rev Immunol 7: 610-621, 2007.

13. Torres-Aguilar H, Aguilar-Ruiz SR, González-Pérez G, Munguía R, Bajaña S, Meraz-Ríos MA and Sánchez-Torres C: Tolerogenic dendritic cells generated with different immunosuppressive cytokines induce antigen-specific anergy and regulatory properties in memory CD4+ T cells. J Immunol 184: 1765-1775, 2010.

14. Fu J, Zhang A and Ju X: Tolerogenic dendritic cells as a target for the therapy of immune thrombocytopenia. Clin Appl Thromb Hemost 18: 469-475, 2012.

15. Ning B, Wei J, Zhang A, Gong W, Fu J, Jia T and Yang SY: Antigen-specific tolerogenic dendritic cells ameliorate the severity of murine collagen-induced arthritis. PLoS One 10: e0131152, 2015.

16. Ragab AA, Van De Motter R, Lavish SA, Goldberg VM, Ninomiya JT, Carlin CR and Greenfield EM: Measurement and removal of adherent endotoxin from titanium particles and implant surfaces. J Orthop Res 17: 803-809, 1999.

17. Vallés G, Gil-Garay E, Munuera L and Vilaboa N: Modulation of the cross-talk between macrophages and osteoblasts by titanium-based particles. Biomaterials 29: 2326-2335, 2008.

18. Zhang M, Tang H, Guo Z, An H, Zhu X, Song W, Guo J, Huang X, Chen T, Wang J and Cao X: Splenic stroma drives mature dendritic cells to differentiate into regulatory dendritic cells. Nat Immunol 5: 1124-1133, 2004.

19. Yang SY, Ren W, Park Y, Sieving A, Hsu S, Nasser S and Wooley PH: Diverse cellular and apoptotic responses to variant shapes of UHMWPE particles in a murine model of inflammation. Biomaterials 23: 3535-3543, 2002.

20. Wooley PH, Morren R, Andary J, Sud S, Yang SY, Mayton L, Markel D, Sieving A and Nasser S: Inflammatory responses to orthopaedic biomaterials in the murine air pouch. Biomaterials 23: 517-526, 2002.

21. Wong RH, Wei JC, Huang CH, Lee HS, Chiou SY, Lin SH, Cai YW, Hung PH, Wang MF and Yang SF: Association of IL-12B genetic polymorphism with the susceptibility and disease severity of ankylosing spondylitis. J Rheumatol 39: 135-1340, 2012.

22. Liu S, Virdi AS, Sena K and Sumner DR: Sclerostin antibody prevents particle-induced implant loosening by stimulating bone formation and inhibiting bone resorption in a rat model. Arthritis Rheum 64: 4012-4020, 2012.

23. de Avila ED, Lima BP, Sekiya T, Torii Y, Ogawa T, Shi W and Lux R: Effect of UV-photofunctionalization on oral bacterial attachment and biofilm formation to titanium implant material. Biomaterials 67: 84-92, 2015.

24. Wei J, Richbourgh B, Jia T and Liu C: ADAMTS-12: A multifaced metalloproteinase in arthritis and inflammation. Mediators Inflamm 2014: 649718, 2014. 
25. Zhang A, Fu J, Ning B, Li D, Sun N, Wei W, Wei J and Ju X: Tolerogenic dendritic cells generated with IL-10/TGF $\beta 1$ relieve immune thrombocytopenia in mice. Thromb Res 132: 63-68, 2013.

26. Lee KH, Abas F, Mohamed Alitheen NB, Shaari K, Lajis NH, Israf DA and Syahida A: Chemopreventive effects of a curcumin-like diarylpentanoid [2,6-bis(2,5-dimethoxybenzylidene)cyclohexanone] in cellular targets of rheumatoid arthritis in vitro. Int J Rheum Dis 18: 616-627, 2015.

27. Zhou F, Mei J, Han X, Li H, Yang S, Wang M, Chu L, Qiao H and Tang T: Kinsenoside attenuates osteoarthritis by repolarizing macrophages through inactivating NF- $\mathrm{BB} / \mathrm{MAPK}$ signaling and protecting chondrocytes. Acta Pharm Sin B 9: 973-985, 2019.
28. Wei X, Zhang X, Flick LM, Drissi H, Schwarz EM and O'Keefe RJ: Titanium particles stimulate COX-2 expression in synovial fibroblasts through an oxidative stress-induced, calpain-dependent, NF-kappaB pathway. Am J Physiol Cell Physiol 297: C310-C320, 2009.

(i) $\Theta$ This work is licensed under a Creative Commons Attribution-NonCommercial-NoDerivatives 4.0 International (CC BY-NC-ND 4.0) License. 